\title{
Organ donation after controlled cardiocirculatory death: confidence by clarity
}

\author{
Thomas Bein $^{1 *} \mathbb{E}$, Alain Combes ${ }^{2}$ and Geert Meyfroidt ${ }^{3}$
}

(c) 2021 The Author(s)

Even if an impressive number of 6.337 organs from 1837 donors were transplanted in the Eurotransplant region in 2020 (a reduction of 667 donors compared to 2019), at the end of the year, the active waiting list remains high, with 14,020 patients (of whom 3502 on the waiting list for heart, lung, or liver transplantation) still in need of an organ upon which their lives depend [1]. Historically, organ donation from deceased donors was possible when they had explicitly expressed the will to donate and were determined to be brain dead, defined as the complete and irreversible loss of all brain functions, but this concept may reveal structural deficiency (recognition and reporting of potential donors, realization of donations) [2]. Ethical evolutions and new scientific insights have changed policies since these early years of cadaver organ donations. First, some countries, such as Austria, Belgium, the Netherlands, and Spain, now have presumed consent or opt-out (instead of opt-in) donor legislations, and Ireland is expected to implement such a legislation soon. These countries typically have high donor rates. In ethical considerations of opt-in or opt-out regulations, important reflections on the weighting of the principles of autonomy, benevolence, solidarity and liberty of the individual are inherent. But whatever the system chosen, trust must be generated by adequate and fair communication [3]. Second, in patients who do not meet formal brain death criteria, donation after cardiac or cardiocirculatory death (DCCD), previously known as 'non-heart-beating' donation, is an alternative option. This can be performed following unsuccessful resuscitation in an uncontrolled DCCD protocol but is more frequently done in the

\footnotetext{
*Correspondence: thomas.bein@ukr.de

${ }^{1}$ Faculty of Medicine, University of Regensburg, Regensburg, Germany

Full author information is available at the end of the article
}

context of controlled DCCD (cDCCD) following withdrawal of life-sustaining therapies (WLST) [4].

The procedure for CDCCD is as follows: after cessation of systemic circulation, and a short standoff time (various definitions due to different national laws) to exclude autoresuscitation, the patient is declared dead according to cardiovascular criteria and can become an organ donor. The dead donor rule is strictly respected. Nowadays, several techniques for organ preservation, either in situ, ex situ, or with machine perfusion, exist and allow for good quality of transplantable organs, and overall good results in the transplanted recipient [5]. During such procedures of post mortem organ recovery a strict focus must be given by monitoring to exclude brain reperfusion [6]. Some countries still do not allow cDCCD due to legal and ethical concerns [7]. To address these concerns, carefully addressed guidelines, written with scientific, legal, and ethical expertise, are necessary, and should cover the timing of determination of death, the issue of proper consent, the role of surrogate decision makers, the preservation of dignity of the dying patient, as well as organ preservation measures [8].

In the current issue of Intensive Care Medicine, Domínguez-Gil et al. have published a Collaborative Statement of an international expert group regarding the management of CDCCD [9]. Their statement aims to clarify some of the clinical, ethical, legal, and practical aspects of this procedure, and will hopefully contribute to a broader acceptance. The importance of such precise statements, born in a process involving multiple iterations, cannot be underestimated in the current situation of donor organ shortage and the many different legislations and practices across Europe. The statement focuses thoroughly on the specific challenges of determining a prognosis that justifies WLST, as well as on specific aspects of the determination of death in this scenario, and measures of perfusion maintenance to optimize

\section{实 Springer}


the function of transplantable organs. The manuscript and statements will appeal not only to those colleagues involved in the care of transplant patients, but to the entire intensive care community [10]. In a clear and concise way, these statements promote transparency and offer practical guidance for the $\mathrm{CDCCD}$ procedure, in the context of controlled withdrawal of therapeutic support, which should be part of daily practice in intensive care regardless of donation perspectives.

However, even if such broad consensus guidelines [9] are an important and necessary step to address the controversies surrounding the DCD procedure, their publication and broad dissemination will not automatically solve all problems in the context of CDCCD. It is clear that the transition from a dying patient who has experienced a cardiac arrest and is undergoing resuscitative efforts for organ preservation touches essential questions on life and death, and mistrust might be fostered without a clear, public discussion and matched transparency (Fig. 1). James F. Childress, co-author of the landmark book Principles of Biomedical Ethics and a leading figure in the field of contemporary bioethics, acknowledges that some health care professionals and institutions continue to suffer from ongoing conflicts of obligation, loyalty, and interests in the context of donation after circulatory death [11]. As argued by Jessie Cooper [12], not all these concerns can be reduced to abstract directives for practice or should be dealt with as an organisational problem. Even if the overall benefit of organ donation after circulatory death is clear, this may not automatically diminish the challenges of a specific care for dying patients-quasi in a utilitarian manner [13]. Furthermore, Childress raises an additional point which may not be sufficiently addressed by statements: "Trust in the healthcare system is the prime consideration" [11]. Such trust can only be established by an open and transparent public debate, where medical and ethical domain experts have a crucial role. In addition, all stakeholders, including patients, and political decision makers should be involved as well. Due to nation's histories as well as cultural/religious beliefs and values and existing frameworks in some countries, like Germany 'Non-heart-beating donors are ineligible' [14] at the moment. Maybe health politics, other stakeholders and the affected people can find inspiration here and should look at those countries where cDCCD is a widely accepted practice. An ethically careful and responsible practice of $\mathrm{CDCCD}$ will only be guaranteed when this measure is 'deeply embedded with specific organisational settings' [12]. In this way, the statements and algorithm by Domínguez-Gil et al. [9] are important further steps to promote CDCCD as a normal endof-life practice for eligible potential donors. Moreover, and even broader, they are an important contribution

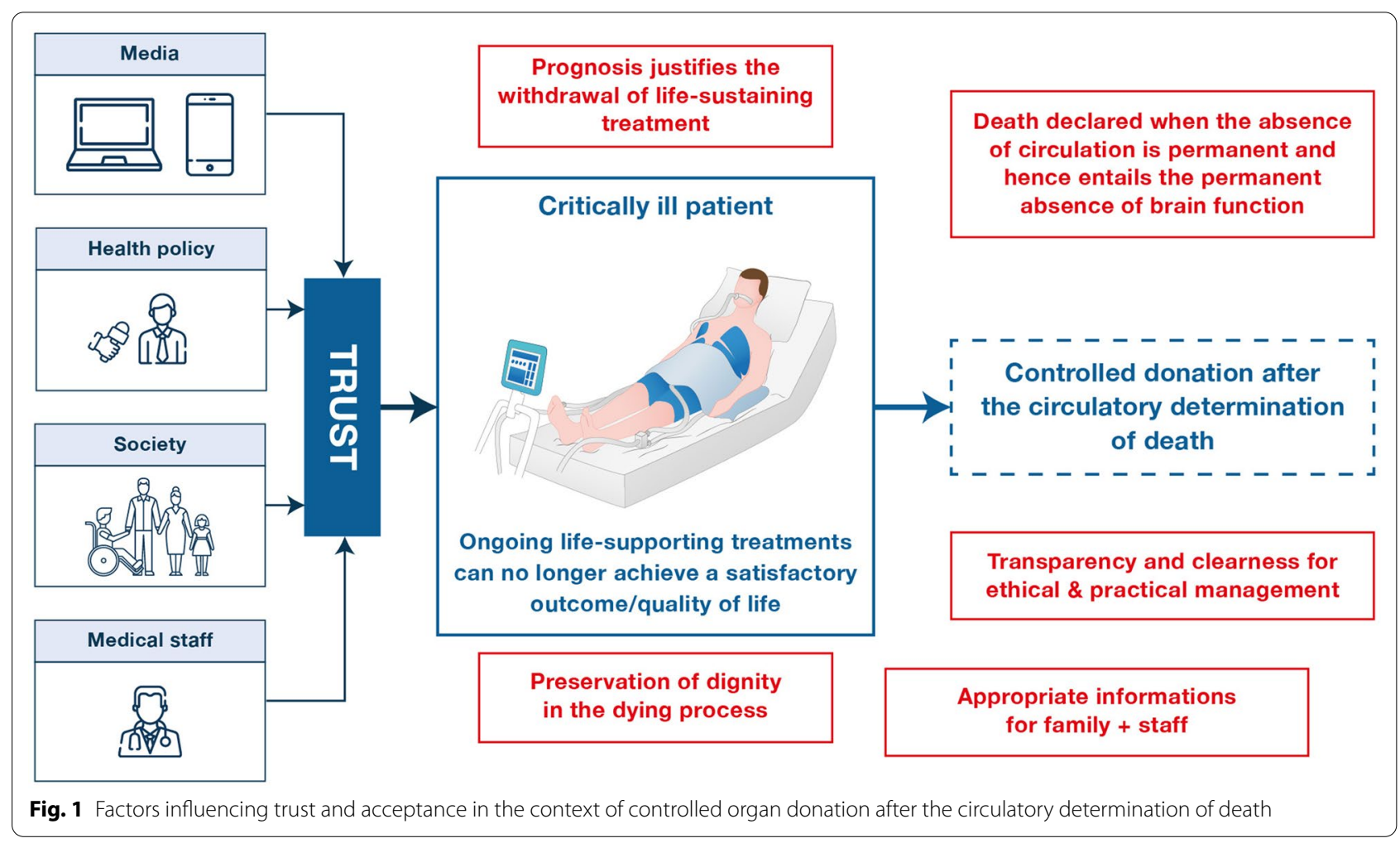


towards the general societal acceptance of this end-oflife challenging procedure.

\author{
Author details \\ ${ }^{1}$ Faculty of Medicine, University of Regensburg, Regensburg, Germany. ${ }^{2}$ Ser- \\ vice de Médecine Intensive-Réanimation Institut de Cardiologie, Hôpital Pitié- \\ Salpêtrière Assistance Publique-Hôpitaux de Paris, APHP Sorbonne Université, \\ INSERM-UMRS 1166, iCAN Institute of Cardiometabolism/Nutrition, 47 bd de \\ I'Hôpital, 75013 Paris, France. ${ }^{3}$ UZ Leuven, campus Gasthuisberg, Herestraat \\ 49, 3000 Leuven, Belgium.
}

\section{Funding}

Open Access funding enabled and organized by Projekt DEAL.

\section{Compliance with ethical standards}

\section{Conflicts of interest}

All authors declare no conflicts of interest.

\section{Open Access}

This article is licensed under a Creative Commons Attribution-NonCommercial 4.0 International License, which permits any non-commercial use, sharing, adaptation, distribution and reproduction in any medium or format, as long as you give appropriate credit to the original author(s) and the source, provide a link to the Creative Commons licence, and indicate if changes were made. The images or other third party material in this article are included in the article's Creative Commons licence, unless indicated otherwise in a credit line to the material. If material is not included in the article's Creative Commons licence and your intended use is not permitted by statutory regulation or exceeds the permitted use, you will need to obtain permission directly from the copyright holder. To view a copy of this licence, visit http://creativecommons.org/licen ses/by-nc/4.0/.

\section{Publisher's Note}

Springer Nature remains neutral with regard to jurisdictional claims in published maps and institutional affiliations.

Received: 15 January 2021 Accepted: 28 January 2021 Published online: 23 February 2021
References

1. https://statistics.eurotransplant.org/index.php?search_type=overv iew\&search_text=9023. Assessed 25 Jan 2021

2. Kutsogiannis DJ, Asthana S, Townsend DR, Singh G, Karvellas CJ (2013) The incidence of potential missed organ donors in intensive care units and emergency rooms: a retrospective cohort. Intensive Care Med 39:1452-1459

3. Beloucif S (2012) Opt-in or opt-out for organtransplantation. Curr Opin Anaesthesiol 25:199-203

4. Smith M, Domínguez-Gil B, Greer DM, Manara AR, Souter MJ (2019) Organ donation after circulatory death: current status and future potential. Intensive Care Med 45:310-321

5. Cypel M, Neyrinck A, Machuca TN (2019) Ex vivo perfusion techniques: state of the art and potential applications. Intensive Care Med 45(3):354356. https://doi.org/10.1007/s00134-019-05568-3

6. Manara A, Shemie SD, Large S et al (2020) Maintaining the permanence principle for death during in situ normothermic regional perfusion for donation after circulatory death organ recovery: a United Kingdom and Canadian proposal. Am J Transplant 20:2017-2025

7. Lomero M, Gardiner D, Coll E et al (2020) Donation after circulatory death today: an updated overview of the European landscape. Transpl Int 33:76-88. https://doi.org/10.1111/tri.13506

8. Bos MA (2005) Ethical and legal Issues in non-heart-beating organ donation. Transplant Proceed 37:574-576

9. Domínguez-Gil B et al (2021) Statement from an International collaborative for expanding controlled donation after the circulatory determination of death. Intensive Care Med. https://doi.org/10.1007/s00134-02006341-7

10. Manara A, Procaccio F, Domínguez-Gil B (2019) Expanding the pool of deceased organ donors: the ICU and beyond. Intensive Care Med 45(3):357-360. https://doi.org/10.1007/s00134-019-05546-9

11. Childress JF (2008) Organ donation after circulatory determination of death: lessons and unresolved controversies. J Law Med Ethics Health Care Winter 2008:766-771

12. Cooper J (2018) Organs and organisations: situating ethics in organ donation after circulatory death in the UK. Soc Sci Med 209:104-110

13. Streat S (2004) Clinical review: moral assumptions and the process of organ donation in the intensive care unit. Crit Care 8:382

14. Heide W (2016) Non-heart-beating donors are ineligible. Der Nervenarzt 87:161-168 